Network Working Group

Request for Comments: 2439

Category: Standards Track
C. Villamizar

ANS

R. Chandra

Cisco

R. Govindan

IS I

November 1998

\title{
BGP Route Flap Damping
}

Status of this Memo

This document specifies an Internet standards track protocol for the Internet community, and requests discussion and suggestions for improvements. Please refer to the current edition of the "Internet Official Protocol Standards" (STD 1) for the standardization state and status of this protocol. Distribution of this memo is unlimited.

Copyright Notice

Copyright (C) The Internet Society (1998). All Rights Reserved.

Abstract

A usage of the BGP routing protocol is described which is capable of reducing the routing traffic passed on to routing peers and therefore the load on these peers without adversely affecting route convergence time for relatively stable routes. This technique has been implemented in commercial products supporting BGP. The technique is also applicable to IDRP.

The overall goals are:

- to provide a mechanism capable of reducing router processing load caused by instability

o in doing so prevent sustained routing oscillations

- to do so without sacrificing route convergence time for generally well behaved routes.

This must be accomplished keeping other goals of BGP in mind:

- pack changes into a small number of updates

- preserve consistent routing 
- minimal addition space and computational overhead

An excessive rate of update to the advertised reachability of a subset of Internet prefixes has been widespread in the Internet. This observation was made in the early 1990s by many people involved in Internet operations and remains the case. These excessive updates are not necessarily periodic so route oscillation would be a misleading term. The informal term used to describe this effect is "route flap". The techniques described here are now widely deployed and are commonly referred to as "route flap damping".

1 Overview

To maintain scalability of a routed internet, it is necessary to reduce the amount of change in routing state propagated by BGP in order to limit processing requirements. The primary contributors of processing load resulting from BGP updates are the BGP decision process and adding and removing forwarding entries.

Consider the following example. A widely deployed BGP implementation may tend to fail due to high routing update volume. For example, it may be unable to maintain it's BGP or IGP sessions if sufficiently loaded. The failure of one router can further contribute to the load on other routers. This additional load may cause failures in other instances of the same implementation or other implementations with a similar weakness. In the worst case, a stable oscillation could result. Such worse cases have already been observed in practice.

A BGP implementation must be prepared for a large volume of routing traffic. A BGP implementation cannot rely upon the sender to sufficiently shield it from route instabilities. The guidelines here are designed to prevent sustained oscillations, but do not eliminate the need for robust and efficient implementations. The mechanisms described here allow routing instability to be contained at an AS border router bordering the instability.

Even where BGP implementations are highly robust, the performance of the routing process is limited. Limiting the propagation of unnecessary change then becomes an issue of maintaining reasonable route change convergence time as a routing topology grows.

2 Methods of Limiting Route Advertisement

Two methods of controlling the frequency of route advertisement are described here. The first involves fixed timers. The fixed timer technique has no space overhead per route but has the disadvantage of slowing route convergence for the normal case where a route does not have a history of instability. The second method overcomes this 
limitation at the expense of maintaining some additional space overhead. The additional overhead includes a small amount of state per route and a very small processing overhead.

It is possible and desirable to combine both techniques. In practice, fixed timers have been set to very short time intervals and have proven useful to pack routes into a smaller number of updates when routes arrive in separate updates. The BGP protocol refers to this as packing Network Layer Reachability Information (NLRI) [5].

Seldom are fixed timers set to the tens of minutes to hours that would be necessary to actually damp route flap. To do so would produce the undesirable effect of severely limiting routing convergence.

\subsection{Existing Fixed Timer Recommendations}

BGP-3 does not make specific recommendations in this area [1]. The short section entitled "Frequency of Route Selection" simply recommends that something be done and makes broad statements regarding certain properties that are desirable or undesirable.

BGP 4 retains the "Frequency of Route Advertisement" section and adds a "Frequency of Route Origination" section. BGP-4 describes a method of limiting route advertisement involving a fixed (configurable)

MinRouteAdvertisementInterval timer and fixed

MinASOriginationInterval timer [5]. The recommended timer values of MinRouteAdvertisementInterval is 30 seconds and

MinASOriginationInterval is 15 seconds.

\subsection{Desirable Properties of Damping Algorithms}

Before describing damping algorithms the objectives need to be clearly defined. Some key properties are examined to clarify the design rationale.

The overall objective is to reduce the route update load without limiting convergence time for well behaved routes. To accomplish this, criteria must be defined for well behaved and poorly behaved routes. An algorithm must be defined which allows poorly behaved routes to be identified. Ideally, this measure would be a prediction of the future stability of a route.

Any delay in propagation of well behaved routes should be minimal. Some delay is tolerable to support better packing of updates. Delay of poorly behave routes should, if possible, be proportional to a measure of the expected future instability of the route. Delay in propagating an unstable route should cause the unstable route to be 
suppressed until there is some degree of confidence that the route has stabilized.

If a large number of route changes are received in separate updates over some very short period of time and these updates have the potential to be combined into a single update then these should be packed as efficiently as possible before propagating further. Some small delay in propagating well behaved routes is tolerable and is necessary to allow better packing of updates.

Where routes are unstable, use and announcement of the routes should be suppressed rather than suppressing their removal. Where one route to a destination is stable, and another route to the same destination is somewhat unstable, if possible, the unstable route should be suppressed more aggressively than if there were no alternate path.

Routing consistency within an AS is very important. Only very minimal delay of internal BGP (IBGP) should be done. Routing consistency across AS boundaries is also very important. It is highly undesirable to advertise a route that is different from the route that is being used, except for a very minimal time. It is more desirable to suppress the acceptance of a route (and therefore the use of that route in the IGP) rather than suppress only the redistribution.

It is clearly not possible to accurately predict the future stability of a route. The recent history of stability is generally regarded as a good basis for estimating the likelihood of future stability. The criteria that is used to distinguish well behaved from poorly behaved routes is therefore based on the recent history of stability of the route. There is no simple quantitative expression of recent stability so a figure of merit must be defined. Some desirable characteristics of this figure of merit would be that the farther in the past that instability occurred, the less it's affect on the figure of merit and that the instability measure would be cumulative rather than reflecting only the most recent event.

The algorithms should behave such that for routes which have a history of stability but make a few transitions, those transitions should be made quickly. If transitions continue, advertisement of the route should be suppressed. There should be some memory of prior instability. The degree to which prior instability is considered should be gradually reduced as long as the route remains announced and stable. 


\subsection{Design Choices}

After routes have been accepted their readvertisement will be briefly suppressed to improve packing of updates. There may be a lengthy suppression of the acceptance of an external route. How long a route will be suppressed is based on a figure of merit that is expected to be correlated to the probability of future instability of a route. Routes with high figure of merit values will be suppressed. An exponential decay algorithm was chosen as the basis for reducing the figure of merit over time. These choices should be viewed as suggestions for implementation.

An exponential decay function has the property that previous instability can be remembered for a fairly long time. The rate at which the instability figure of merit decays slows as time goes on. Exponential decay has the following property.

$$
f(f(f i g u r e-o f-m e r i t, t 1), t 2)=f(f i g u r e-o f-m e r i t, t 1+t 2)
$$

This property allows the decay for a long period to be computed in a single operation regardless of the current value (figure-of-merit). As a performance optimization, the decay can be applied in fixed time increments. Given a desired decay half life, the decay for a single time increment can be computed ahead of time. The decay for multiple time increments is expressed below.

$$
f(f i g u r e-o f-m e r i t, \quad n * t 0)=f(f i g u r e-o f-m e r i t, t 0) * \star n=K * \star n
$$

The values of $K$ ** $n$ can be precomputed for a reasonable number of "n" and stored in an array. The value of "K" is always less than one. The array size can be bounded since the value quickly approaches zero. This makes the decay easy to compute using an array bound check, an array lookup and a single multiply regardless as to how much time has elapsed.

3 Limiting Route Advertisements using Fixed Timers

This method of limiting route advertisements involves the use of fixed timers applied to the process of sending routes. It's primary purpose is to improve the packing of routes in BGP update messages. The delay in advertising a stable route should be bounded and minimal. The delay in advertising an unreachable need not be zero, but should also be bounded and should probably have a separate bound set less than or equal to the bound for a reachable advertisement.

The BGP protocol defines the use of a Routing Information Base (RIB). Routes that need to be readvertised can be marked in the RIB or an external set of structures maintained, which references the RIB. 
Periodically, a subset of the marked routes can be flushed. This is fairly straightforward and accomplishes the objectives. Computation for too simple an implementation may be order $\mathrm{N}$ squared. To avoid $\mathrm{N}$ squared performance, some form of data structure is needed to group routes with common attributes.

An implementation should pack updates efficiently, provide a minimum readvertisement delay, provide a bounds on the maximum

readvertisement delay that would be experienced solely as a result of the algorithm used to provide a minimum delay, and must be computationally efficient in the presence of a very large number of candidates for readvertisement.

4 Stability Sensitive Suppression of Route Advertisement

This method of limiting route advertisements uses a measure of route stability applied on a per route basis. This technique is applied when receiving updates from external peers only (EBGP). Applying this technique to IBGP learned routes or to advertisement to IBGP or EBGP peers after making a route selection can result in routing loops.

A figure of merit based on a measure of instability is maintained on a per route basis. This figure of merit is used in the decision to suppress the use of the route. Routes with high figure of merit are suppressed. Each time a route is withdrawn, the figure of merit is incremented. While the route is not changing the figure of merit value is decayed exponentially with separate decay rates depending on whether the route is stable and reachable or has been stable and unreachable. The decay rate may be slower when the route is unreachable, or the stability figure of merit could remain fixed (not decay at all) while the route remains unreachable. Whether to decay unreachable routes at the same rate, a slower rate, or not at all is an implementation choice. Decaying at a slower rate is recommended.

A very efficient implementation is suggested in the following sections. The implementation only requires computation for the routes contained in an update, when an update is received or withdrawn (as opposed to the simplistic approach of periodically decaying each route). The suggested implementation involves only a small number of simple operations, and can be implemented using scaled integers.

The behavior of unstable routes is fairly predictable. Severely flapping routes will often be advertised and withdrawn at regular time intervals corresponding to the timers of a particular protocol (the IGP or exterior protocol in use where the problem exists). Marginal circuits or mild congestion can result in a long term pattern of occasional brief route withdrawal or occasional brief 
connectivity.

\subsection{Single vs. Multiple Configuration Parameter sets}

The behavior of the algorithm is modified by a number of configurable parameters. It is possible to configure separate sets of parameters designed to handle short term severe route flap and chronic milder route flap (a pattern of occasional drops over a long time period). The former would require a fast decay and low threshold (allowing a small number of consecutive flaps to cause a route to be suppressed, but allowing it to be reused after a relatively short period of stability). The latter would require a very slow decay and a higher threshold and might be appropriate for routes for which there was an alternate path of similar bandwidth.

It may also be desirable to configure different thresholds for routes with roughly equivalent alternate paths than for routes where the alternate paths have a lower bandwidth or tend to be congested. This can be solved by associating a different set of parameters with different ranges of preference values. Parameter selection could be based on BGP LOCAL_PREF.

Parameter selection could also be based on whether an alternate route was known. A route would be considered if, for any applicable parameter set, an alternate route with the specified preference value existed and the figure of merit associated with the parameter set did not indicate a need to suppress the route. A less aggressive suppression would be applied to the case where no alternate route at all existed. In the simplest case, a more aggressive suppression would be applied if any alternate route existed. Only the highest preference (most preferred) value needs to be specified, since the ranges may overlap.

It might also be desirable to configure a different set of thresholds for routes which rely on switched services and may disconnect at times to reduce connect charges. Such routes might be expected to change state somewhat more often, but should be suppressed if continuous state changes indicate instability.

While not essential, it might be desirable to be able to configure multiple sets of configuration parameters per route. It may also be desirable to be able to configure sets of parameters that only correspond to a set of routes (identified by AS path, peer router, specific destinations or other means). Experience may dictate how much flexibility is needed and how to best to set the parameters. Whether to allow different damping parameter sets for different routes, and whether to allow multiple figures of merit per route is an implementation choice. 
Parameter selection can also be based on prefix length. The rationale is that longer prefixes tend to reach less end systems and are less important and these less important prefixes can be damped more aggressively. This technique is in fairly widespread use. Small sites or those with dense address allocation who are multihomed are often reachable by long prefixes which are not easily aggregated. These sites tend to dispute the choice of prefix length for parameter selection. Advocates of the technique point out that it encourages better aggregation.

\subsection{Configuration Parameters}

At configuration time, a number of parameters may be specified by the user. The configuration parameters are expressed in units meaningful to the user. These differ from the parameters used at run time which are in unit convenient for computation. The run time parameters are derived from the configuration parameters. Suggested configuration parameters are listed below.

cutoff threshold (cut)

This value is expressed as a number of route withdrawals. It is the value above which a route advertisement will be suppressed.

reuse threshold (reuse)

This value is expressed as a number of route withdrawals. It is the value below which a suppressed route will now be used again.

maximum hold down time ( $\mathrm{T}$-hold)

This value is the maximum time a route can be suppressed no matter how unstable it has been prior to this period of stability.

decay half life while reachable (decay-ok)

This value is the time duration in minutes or seconds during which the accumulated stability figure of merit will be reduced by half if the route if considered reachable (whether suppressed or not).

decay half life while unreachable (decay-ng)

This value is the time duration in minutes or seconds during which the accumulated stability figure of merit will be reduced by half if the route if considered unreachable. If not specified or set to zero, no decay will occur while a route 
remains unreachable.

decay memory limit (Tmax-ok or Tmax-ng)

This is the maximum time that any memory of previous instability will be retained given that the route's state remains unchanged, whether reachable or unreachable. This parameter is generally used to determine array sizes.

There may be multiple sets of the parameters above as described in Section 4.1. The configuration parameters listed below would be applied system wide. These include the time granularity of all computations, and the parameters used to control reevaluation of routes that have previously been suppressed.

time granularity (delta-t)

This is the time granularity in seconds used to perform all decay computations.

reuse list time granularity (delta-reuse)

This is the time interval between evaluations of the reuse lists. Each reuse lists corresponds to an additional time increment.

reuse list memory reuse-list-max

This is the time value corresponding to the last reuse list. This may be the maximum value of T-hold for all parameter sets of may be configured.

number of reuse lists (reuse-list-size)

This is the number of reuse lists. It may be determined from reuse-list-max or set explicitly.

A recommended optimization is described in section 4.8.6 that involves an array referred to as the "reuse index array". A reuse index array is needed for each decay rate in use. The reuse index array is used to estimate which reuse list to place a route when it is suppressed. Proper placement avoids the need to periodically evaluate decay to determine if a route can be reused or when storage can be recovered. Using the reuse index array avoids the need to compute a logarithm to determine placement. One additional system wide parameter can be introduced. 
reuse index array size (reuse-index-array-size)

This is the size of reuse index arrays. This size determines the accuracy with which suppressed routes can be placed within the set of reuse lists when suppressed for a long time.

\subsection{Guidelines for setting Parameters}

The decay half life should be set to a time considerably longer than the period of the route flap it is intended to address. For example, if the decay is set to ten minutes and a route is withdrawn and readvertised exactly every ten minutes, the route would continue to flap if the cutoff was set to a value of 2 or above.

The stability figure of merit itself is an accumulated time decayed total. This must be kept in mind in setting the decay time, cutoff values and reuse values. The figure of merit is increased each time a route transitions from reachable to unreachable. The figure of merit is decayed at a rate proportional to its current value. Increasing the rate of route flap therefore increments the figure of merit more often and reaches a given threshhold in a shorter amount of time. When the response to a constant rate route flap is plotted this looks like a sawtooth with an abrupt rising edge and a decaying falling edge. Since the absolute decay amount is proportional to the figure of merit, at a continuous constant flap rate the baseline of the sawtooth will tend to stop rising and converge if not clipped by a ceiling value.

If clipped by a ceiling value, the sawtooth baseline will simply reach the ceiling faster at a higher rate of route flap. For example, if flapping at four times the decay rate the following progression occurs. When the route becomes unreachable the first time the value becomes 1 . When the next flap occurs, one is added to the previous value, which has been decreased by the fourth root of 2 (the amount of decay that would occur in $1 / 4$ of the half life time if decay is exponential). The sequence is $1,1.84,2.55,3.14,3.64$, $4.06,4.42,4.71,4.96,5.17, \ldots$, converging at about 6.285 . If a route flaps at four times the decay rate, it will reach 3 in 4 cycles, 4 in 6 cycles, 5 in 10 cycles, and will converge at about 6.3. At twice the decay time, it will reach 3 in 7 cycles, and converge at a value of less than 3.5 .

Figure 1 shows the stability figure of merit for route flap at a constant rate. The time axis is labeled in multiples of the decay half life. The plots represent route flap with a period of 1/2, 1/3, 1/4, and $1 / 8$ times the decay half life. A ceiling of 4.5 was set, which can be seen to affect three of the plots, effectively limiting the time it takes to readvertise the route regardless of the prior 
history. With cutoff and reuse thresholds of 1.5 and 0.75 , routes would be suppressed after being declared unreachable 2-3 times and be used again after approximately 2 decay half life periods of stability .

This function can be expressed formally. Reachability of a route can be represented by a variable "R" with possible values of 0 and 1 representing unreachable and reachable. At a discrete time $\mathrm{R}$ can only have one value. The figure of merit is increased by 1 at each transition from $R=1$ to $R=0$ and clipped to a ceiling value. The decay in figure of merit can then be expressed over a set of discrete times as follows.

figure-of-merit $(t)=K \star$ figure-of-merit $(t-$ delta-t)

$\mathrm{K}=\mathrm{K} 1$ for $\mathrm{R}=0 \mathrm{~K}=\mathrm{K} 2$ for $\mathrm{R}=1$

The four plots are presented vertically. Due to space limitations, only a limited set of points along the time axis are shown. The value of the figure of merit is given. Along side each value is a very low resolution strip chart made up of ASCII dots. This is just intended to give a rough feel for the rise and fall of the values. The strip charts are not displayed on an overlapping set of axes because the sawtooth waveforms cross each other quite frequently. At the very low resolution of these plots, the rise and fall of the baseline is evident, but the sawtooth nature is only observed in the printed value.

From the maximum hold time value ( $\mathrm{T}$-hold), a ratio of the reuse value to a ceiling can be determined. An integer value for the ceiling can then be chosen such that overflow will not be a problem and all other values can be scaled accordingly. If both cutoffs are specified or if multiple parameter sets are used the highest ceiling will be used. 


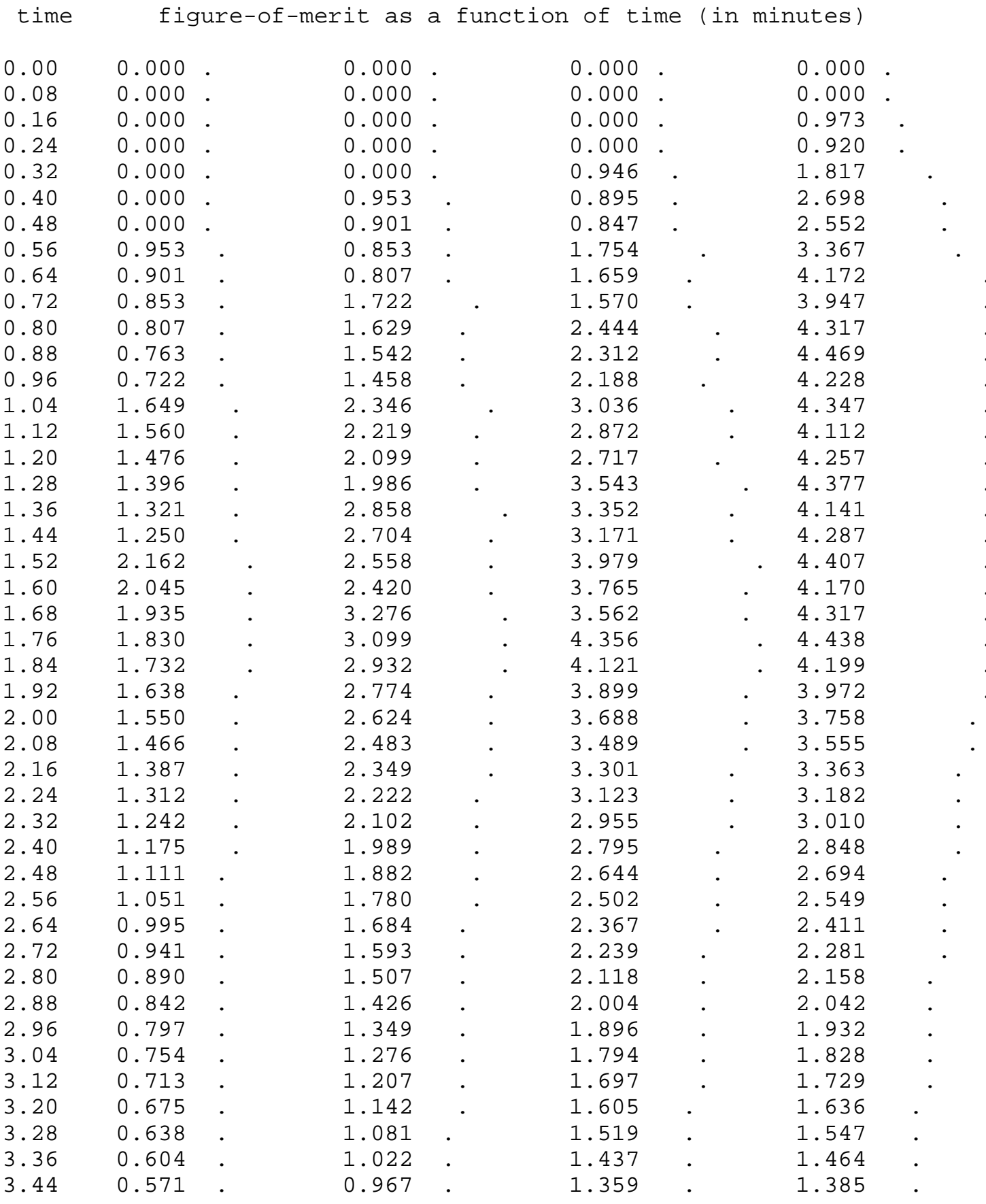

Figure 1: Instability figure of merit for flap at a constant rate 


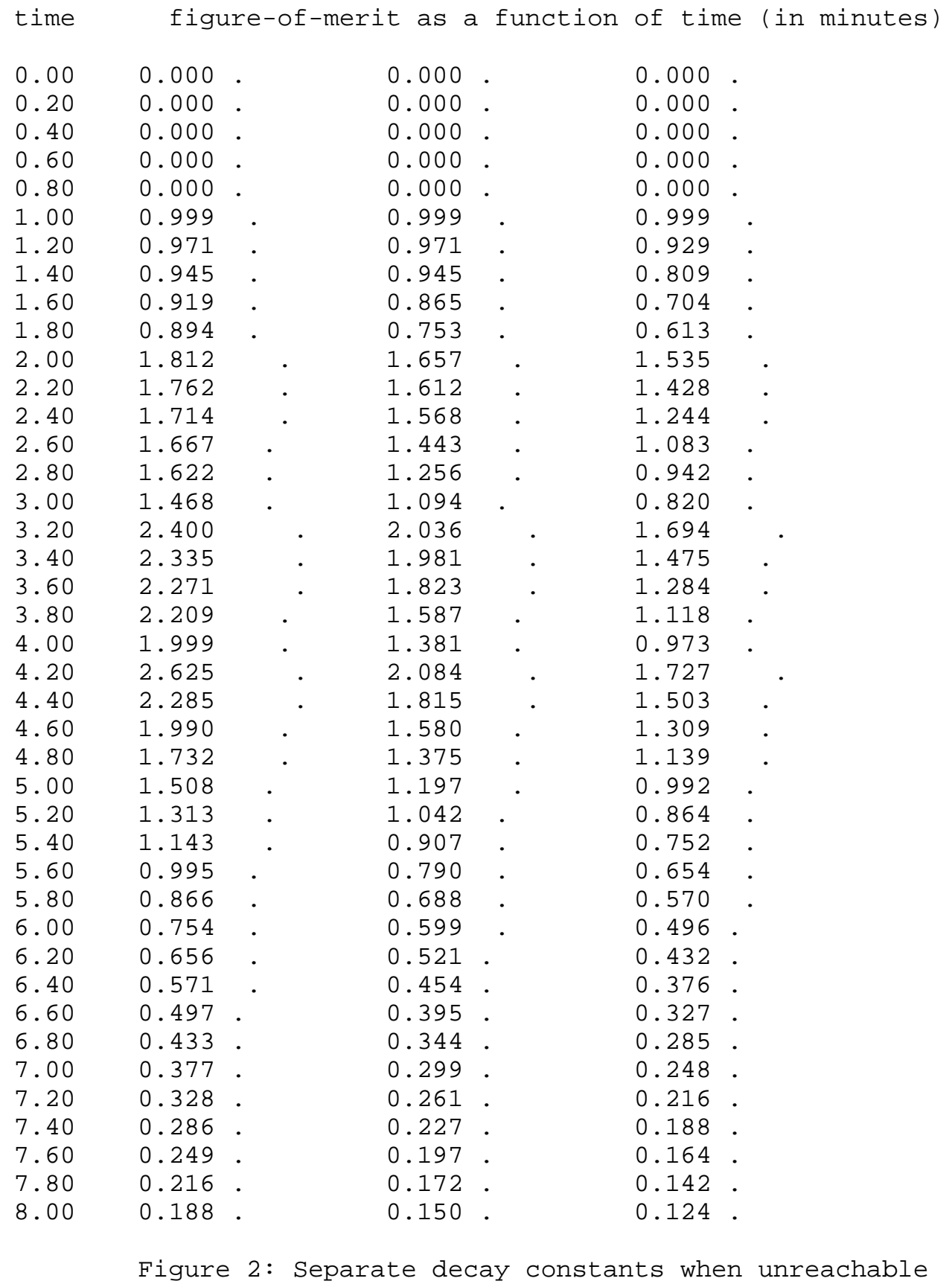


Figure 2 shows the effect of configuring separate decay rates to be used when the route is reachable or unreachable. The decay rate is 5 times slower when the route is unreachable. In the three case shown, the period of the route flap is equal to the decay half life but the route is reachable $1 / 8$ of the time in one, reachable 1/2 the time in one, and reachable $7 / 8$ of the time in the other. In the last case the route is not suppressed until after the third unreachable (when it is above the top threshold after becoming reachable again).

The main point of Figure 2 is to show the effect of changing the duty cycle of the square wave in the variable "R" for a fixed frequency of the square wave. If the decay constants are chosen such that decay is slower when $R=0$ (the route is unreachable), then the figure of merit rises more slowly (more accurately, the baseline of the sawtooth waveform rises more slowly) if the route is reachable a larger percentage of the time. The effect when the route becomes persistently reachable again can be fairly negligible if the sawtooth is clipped by a ceiling value, but is more significant if a slow route flap rate or short interval of route flapping is such that the sawtooth does not reach the ceiling value. In Figure 2 the interval in which the routes are unstable is short enough that the ceiling value is not reached, therefore, the routes that are reachable for a greater percentage of the route flap cycle are reused (placed in the RIB and advertised to peers) sooner than others after the route becomes stable again ("R" becomes 1, indicating the announced state goes to reachable and remains there).

In both Figure 1 and Figure 2, routes would be suppressed. Routes flapping at the decay half life or less would be withdrawn two or three times and then remain withdrawn until they had remained stably announced and stable for on the order of $11 / 2$ to $21 / 2$ times the decay half life (given the ceiling in the example).

The purpose of damping BGP route flap is to reduce the processor burden at the immediate router and the processor burden to downstream routers (BGP peer routers and peers of peers that will see the route announcements advertised by the immediate router). Computing a figure of merit at each discrete time interval using figure-ofmerit $(t)=K$ * figure-of-merit( $t$ - delta-t) would be very inefficient and defeat the purpose. This problem is addressed by defering computation as long as possible and doing a single simple computation to compensate for the decay during the time that has elapsed since the figure of merit was last updated. The use of decay arrays provides the single simple calculation. The use of reuse lists (described later) provide a means to defer calculations. A route becomes usable if there was not further change for a period of time and the route is unreachable. The data structure storage is recovered if the route's state has not changed for a period of time 
and it has been unreachable. The reuse arrays provide a means to estimate how long a computation can be deferred if there is no further change.

A larger time granularity will keep table storage down. The time granularity should be less than a minimal reasonable time between expected worse case route flaps. It might be reasonable to fix this parameter at compile time or set a default and strongly recommend that the user leave it alone. With an exponential decay, array size can be greatly reduced by setting a period of complete stability after which the decayed total will be considered zero rather than retaining a tiny quantity. Alternately, very long decays can be implemented by multiplying more than once if array bounds are exceeded.

The reuse lists hold suppressed routes grouped according to how long it will be before the routes are eligible for reuse. Periodically each list will be advanced by one position and one list removed as described in Section 4.8.7. All of the suppressed routes in the removed list will be reevaluated and either used or placed in another list according to how much additional time must elapse before the route can be reused. The last list will always contain all the routes which will not be advertised for more time than is appropriate for the remaining list heads. When the last list advances to the front, some of the routes will not be ready to be used and will have to be requeued. The time interval for reconsidering suppressed routes and number of list heads should be configurable. Reasonable defaults might be 30 seconds and 64 list heads. A route suppressed for a long time would need to be reevaluated every 32 minutes.

\subsection{Run Time Data Structures}

A fixed small amount of per system storage will be required. Where sets of multiple configuration parameters are used, storage will be required per set of parameters. A small amount of per route storage is required. A set of list heads is needed. These list heads are used to arrange suppressed routes according to the time remaining until they can be reused.

A separate reuse list can be used to hold unreachable routes for the purpose of later recovering storage if they remain unreachable too long. This might be more accurately described as a recycling list. The advantage this would provide is making free data structures available as soon as possible. Alternately, the data structures can simply be placed on a queue and the storage recovered when the route hits the front of the queue and if storage is needed. The latter is less optimal but simple.

Villamizar, et. al. Standards Track [Page 15] 
If multiple sets of configuration parameters are allowed per route, there is a need for some means of associating more than one figure of merit and set of parameters with each route. Building a linked list of these objects seems like one of a number of reasonable implementations. Similarly, a means of associating a route to a reuse list is required. A small overhead will be required for the pointers needed to implement whatever data structure is chosen for the reuse lists. The suggested implementation uses a double linked lists and so requires two pointers per figure of merit.

Each set of configuration parameters can reference decay arrays and reuse arrays. These arrays should be shared among multiple sets of parameters since their storage requirement is not negligible. There will be only one set of reuse list heads for the entire router.

\subsubsection{Data Structures for Configuration Parameter sets}

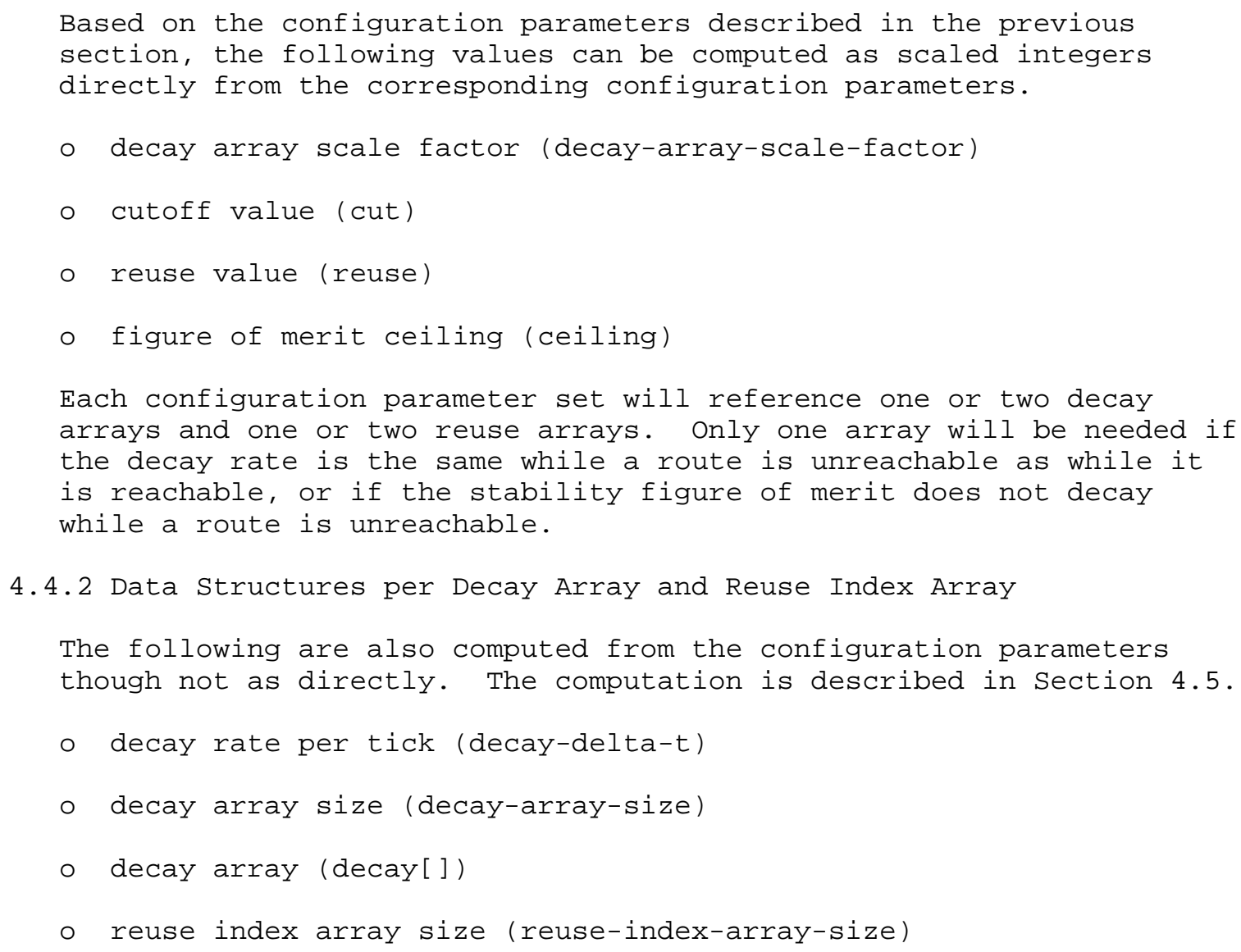


o reuse index array (reuse-index-array[])

For each decay rate specified, an array will be used to store the value of a computed parameter raised to the power of the index of each array element. This is to speed computations. The decay rate per tick is an intermediate value expressed as a real number and used to compute the values stored in the decay arrays. The array size is computed from the decay memory limit configuration parameter expressed as an array size or as a maximum hold time.

The decay array size must be of sufficient size to accommodate the specified decay memory given the time granularity, or sufficient to hold the number of array elements until integer rounding produces a zero result if that value is smaller, or a implementation imposed reasonable size to prevent configurations which use excessive memory. Implementations may chose to make the array size shorter and multiply more than once when decaying a long time interval to reduce storage.

The reuse index arrays serve a similar purpose to the decay arrays. In BGP, a route is said to be "used" if it is considered the best route. In this context, if the route is "used" it is placed in the RIB and is eligible for advertisement to BGP peers. If a route is withdrawn (a BGP announcement is made by a peer indicating that it is no longer reachable), then it is no longer eligible for "use". When a route becomes reachable it may not be "used" immediately if the figure of merit indicates that a recent instability has occurred. After the route remains stable and the figure of merit decays below the "reuse" threshhold, the route is said to be eligible to be "reused" (treated as truly reachable, placed in the RIB and advertised to peers). The amount of time until a route can be reused can be determined using a array lookup. The array can be built given the decay rate. The array is indexed using a scaled integer proportional to the ratio between a current stability figure of merit value and the value needed for the route to be reused.

\subsubsection{Per Route state}

Information must be maintained per some tuple representing a route. At the very minimum, the NLRI (BGP prefix and length) must be contained in the tuple. Different BGP attributes may be included or excluded depending on the specific situation. The AS path should also be contained in the tuple by default. The tuple may also optionally contain other BGP attributes such as MULTI_EXIT_DISCRIMINATOR (MED).

The tuple representing a route for the purpose of route flap damping is:

Villamizar, et. al. Standards Track

[Page 17] 


$\begin{array}{lll}\text { tuple entry } & \text { default } & \text { options } \\ \text { NLRI } & & \\ \quad \text { prefix } & & \\ \text { length } & \begin{array}{l}\text { required } \\ \text { required }\end{array} & \\ \text { AS path } & \text { included } & \text { option to exclude } \\ \text { last AS set in path } & \text { excluded } & \text { option to include } \\ \text { next hop } & \text { excluded } & \text { option to include } \\ \text { MED } & \text { excluded } & \text { option to include } \\ & & \text { in comparisons only }\end{array}$

The AS path is generally included in order to identify downstream instability which is not being damped or not being sufficiently damped and is alternating between a stable and an unstable path. Under rare circumstances it may be desirable to exclude AS path for all or a subset of prefixes. If an AS path ends in an AS set, in practice the path is always for an aggregate. Changes to the trailing AS set should be ignored. Ideally the AS path comparison should insure that at least one AS has remained constant in the old and new AS set, but completely ignoring the contents of a trailing AS set is also acceptable.

Including next hop and MED changes can help suppress the use of an AS which is internally unstable or avoid a next hop which is closer to an unstable IGP path in the adjacent AS. If a large number of MED values are used, the increase in the amount of state may become a problem. For this reason MED is disabled by default and enabled only as part of the tuple comparison, using a single state entry regardless of MED value. Including MED will suppress the use of the adjacent AS even though the change need not be propagated further. Using MED is only a safe practice if a path is known to exist through another AS or where there are enough peering sites with the adjacent AS such that routes heard at only a subset of the peering sites will be suppressed.

\subsubsection{Data Structures per Route}

The following information must be maintained per route. A route here is considered to be a tuple usually containing NLRI, next hop, and AS path as defined in section 4.4.3.

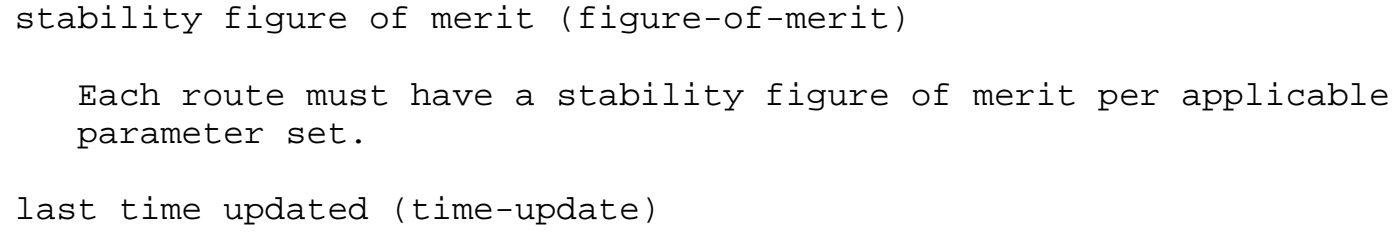


The exact last time updated must be maintained to allow exponential decay of the accumulated figure of merit to be deferred until the route might reasonable be considered eligible for a change in status (having gone from unreachable to reachable or advancing within the reuse lists).

config block pointer

Any implementation that supports multiple parameter sets must provide a means of quickly identifying which set of parameters corresponds to the route currently being considered. For implementations supporting only parameter sets where all routes must be treated the same, this pointer is not required.

reuse list traversal pointers

If doubly linked lists are used to implement reuse lists, then two pointers will be needed, previous and next. Generally there is a double linked list which is unused when a route is suppressed from use that can be used for reuse list traversal eliminating the need for additional pointer storage.

\subsection{Processing Configuration Parameters}

From the configuration parameters, it is possible to precompute a number of values that will be used repeatedly and retain these to speed later computations that will be required frequently.

Scaling is usually dependent on the highest value that figureof-merit can attain, referred to here as the ceiling. The real number value of the ceiling will typically be determined by the following equation. The ceiling can also be configured to a specific value, which in turn dictates T-hold.

$$
\text { ceiling }=\text { reuse } *(\exp (T-h o l d / \text { decay-half-life }) * \log (2))
$$

In the above equation, reuse is the reuse threshhold described in Section 4.2 .

The methods of scaled integer arithmetic are not described in detail here. The methods of determining the real values are given. Translation into scaled integer values and the details of scaled integer arithmetic are left up to the individual implementations.

The ceiling value can be set to be the largest integer that can fit in half the bits available for an unsigned integer. This will allow the scaled integers to be multiplied by the scaled decay 
value and then shifted down. Implementations may prefer to use real numbers or may use any integer scaling deemed appropriate for their architecture.

penalty value and thresholds (as proportional scaled integers)

The figure of merit penalty for one route withdrawal and the cutoff values must be scaled according to the above scaling factor.

decay rate per tick (decay[1])

The decay value per increment of time as defined by the time granularity must be determined (at least initially as a floating point number). The per tick decay is a number slightly less than one. It is the Nth root of the one half where $\mathrm{N}$ is the half life divided by the time granularity.

$$
\operatorname{decay}[1]=\exp ((1 /(\text { decay-half-life/delta-t })) * \log (1 / 2))
$$

decay array size (decay-array-size)

The decay array size is the decay memory divided by the time granularity. If integer truncation brings the value of an array element to zero, the array can be made smaller. An implementation should also impose a maximum reasonable array size or allow more than one multiplication.

$$
\text { decay-array-size }=(\text { Tmax/delta-t })
$$

decay array (decay[])

Each i-th element of the decay array is the per tick delay raised to the i-th power. This might be best done by successive floating point multiplies followed by scaling and integer rounding or truncation. The array itself need only be computed at startup.

$$
\operatorname{decay}[i]=\operatorname{decay}[1] * \star i
$$

\section{6 Building the Reuse Index Arrays}

The reuse lists may be accessed quite frequently if a lot of routes are flapping sufficiently to be suppressed. A method of speeding the determination of which reuse list to use for a given route is suggested. This method is introduced in section 4.2, its configuration described in section 4.4 .2 and the algorithms described in section 4.8.6 and section 4.8.7. This section describes building 
the reuse list index arrays.

A ratio of the figure of merit of the route under consideration to the cutoff value is used as the basis for an array lookup. The ratio is scaled and truncated to an integer and used to index the array. The array entry is an integer used to determine which reuse list to use.

reuse array maximum ratio (max-ratio)

This is the maximum ratio between the current value of the stability figure of merit and the target reuse value that can be indexed by the reuse array. It may be limited by the ceiling imposed by the maximum hold time or by the amount of time that the reuse lists cover.

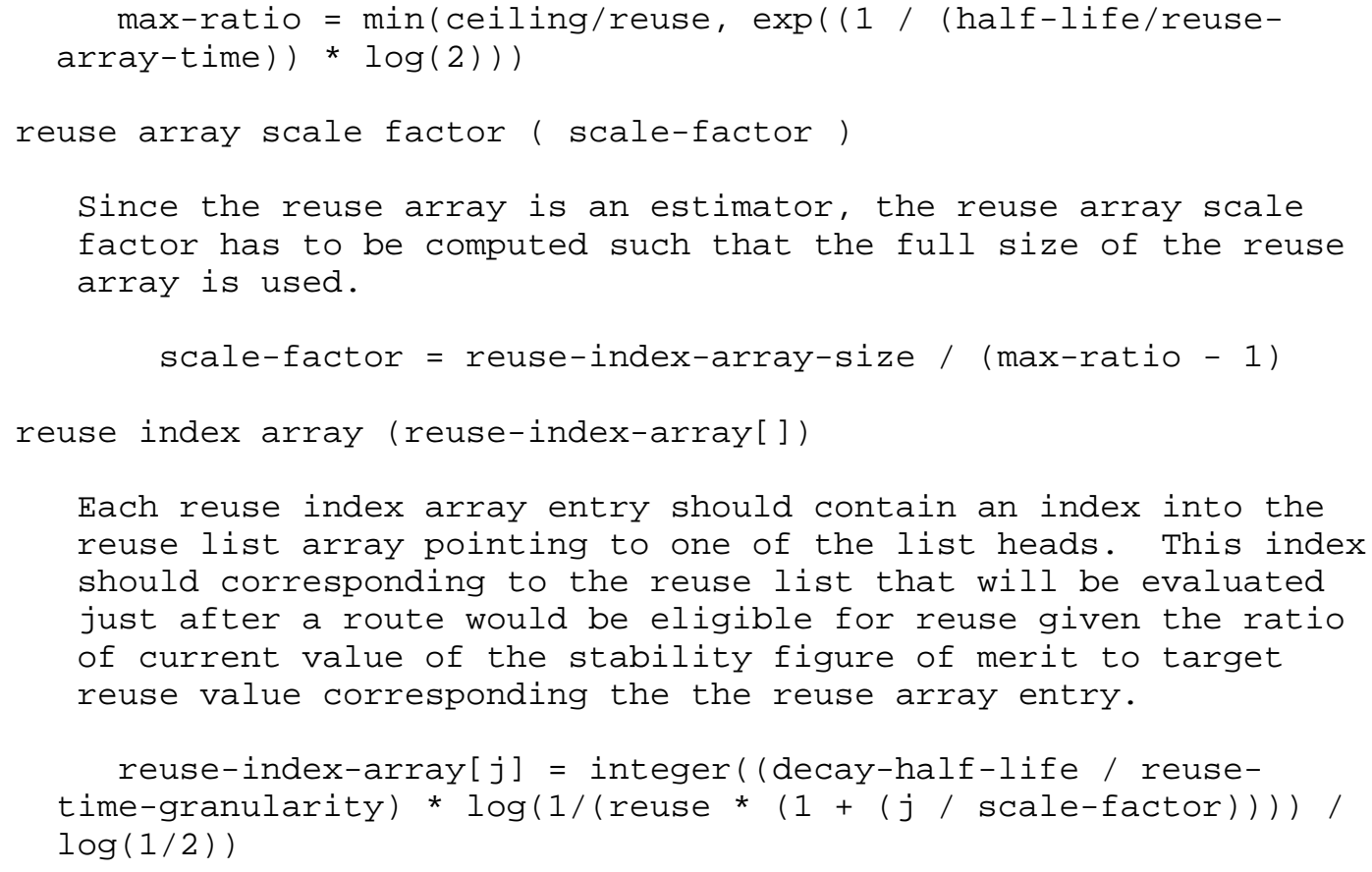

To determine which reuse queue to place a route which is being suppressed, the following procedure is used. Divide the current figure of merit by the cutoff. Subtract one. Multiply by the scale factor. This is the index into the reuse index array (reuse-indexarray[]). The value fetched from the reuse index array (reuseindex-array[]) is an index into the array of reuse lists (reusearray[]). If this index is off the end of the array use the last queue otherwise look in the array and pick the number of the queue 
from the array at that index. This is quite fast and well worth the setup and storage required.

4.7 A Sample Configuration

A simple example is presented here in which the space overhead is estimated for a set of configuration parameters. The design here assumes:

1. there is a single parameter set used for all routes,

2. decay time for unreachable routes is slower than for reachable routes

3. the arrays must be full size, rather than allow more than one multiply per decay operation to reduce the array size.

This example is used in later sections. The use of multiple parameter sets complicates the examples somewhat. Where multiple parameter sets are allowed for a single route, the decay portion of the algorithm is repeated for each parameter set. If different routes are allowed to have different parameter sets, the routes must have pointers to the parameter sets to keep the time to locate to a minimum, but the algorithms are otherwise unchanged.

A sample set of configuration parameters and a sample set of implementation parameters are provided in in the two following lists.

1. Configuration Parameters

o cut $=1.25$

o reuse $=0.5$

o $\mathrm{T}$-hold $=15$ mins

o decay-ok $=5 \mathrm{~min}$

o decay-ng $=15 \mathrm{~min}$

o Tmax-ok, Tmax-ng $=15,30$ mins

2. Implementation Parameters

o delta-t $=1$ sec

- delta-reuse $=15 \mathrm{sec}$ 


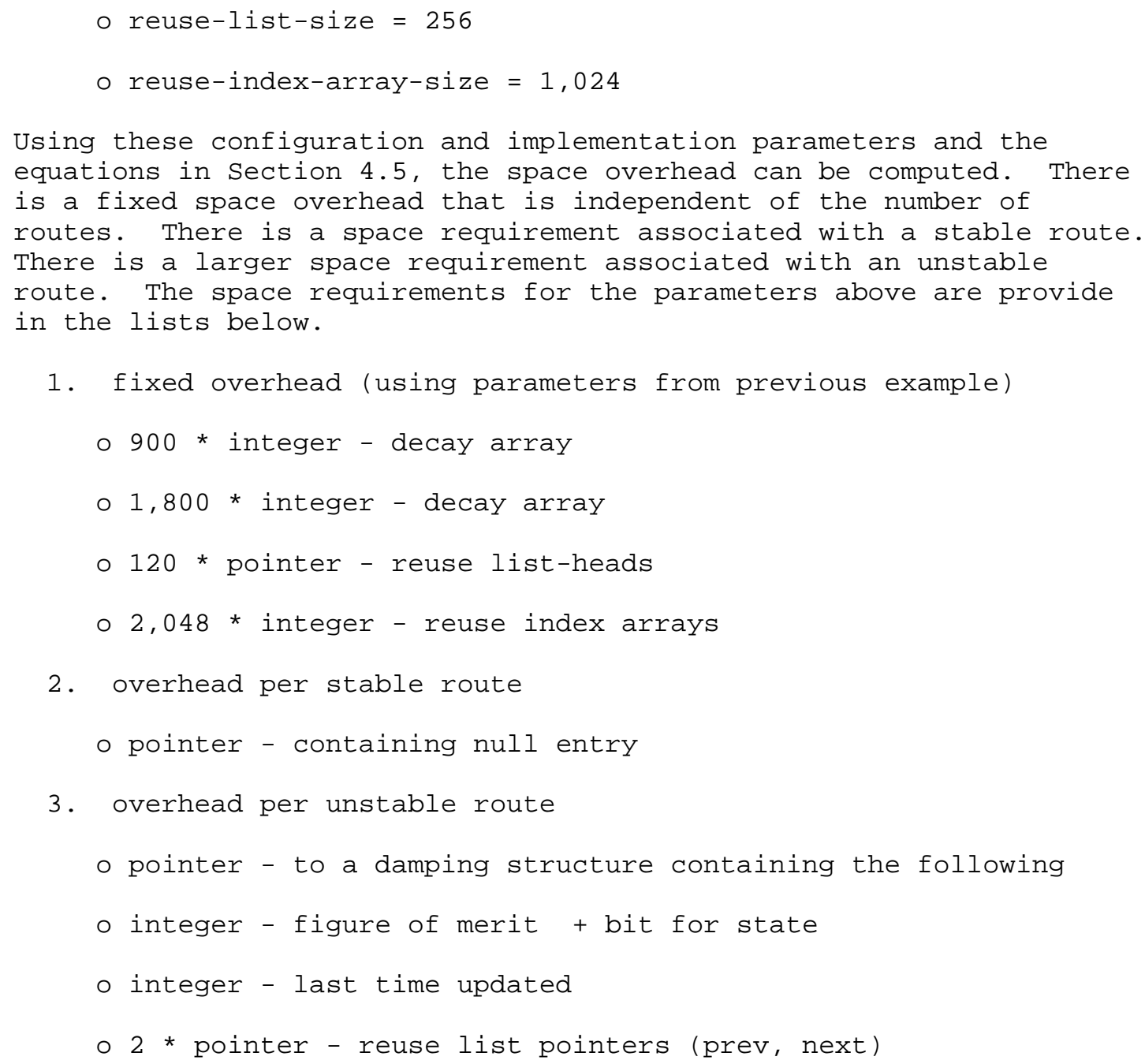

2. overhead per stable route

o pointer - containing null entry

3. overhead per unstable route

o pointer - to a damping structure containing the following

o integer - figure of merit + bit for state

o integer - last time updated

o 2 * pointer - reuse list pointers (prev, next)

The decay arrays are sized acording to delta-t and Tmax-ok or Tmaxng. The number of reuse list-heads is based on delta-reuse and the greater of Tmax-ok or Tmax-ng. There are two reuse index arrays whose size is a configured parameter.

Figure 3 shows the behavior of the algorithm with the parameters given above. Four cases are given in this example. In all four, there is a twelve minute period of route oscillations. Two periods of oscillation are used, 2 minutes and 4 minutes. Two duty cycles are used, one in which the route is reachable during $20 \%$ of the cycle and the other where the route is reachable during $80 \%$ of the cycle. In all four cases, the route becomes suppressed after it becomes 
unreachable the second time. Once suppressed, it remains suppressed until some period after becoming stable. The routes which oscillate over a 4 minute period are no longer suppressed within 9-11 minutes after becoming stable. The routes with a 2 minute period of oscillation are suppressed for nearly the maximum 15 minute period after becoming stable.

\subsection{Processing Routing Protocol Activity}

The prior sections concentrate on configuration parameters and their relationship to the parameters and arrays used at run time and provide the algorithms for initializing run time storage. This section provides the steps taken in processing routing events and timer events when running.

The routing events are:

1. A BGP peer or new route comes up for the first time (or after an extended down time) (Section 4.8.1)

2. A route becomes unreachable (Section 4.8.2)

3. A route becomes reachable again (Section 4.8.3)

4. A route changes (Section 4.8 .4 )

5. A peer goes down (Section 4.8.5) 


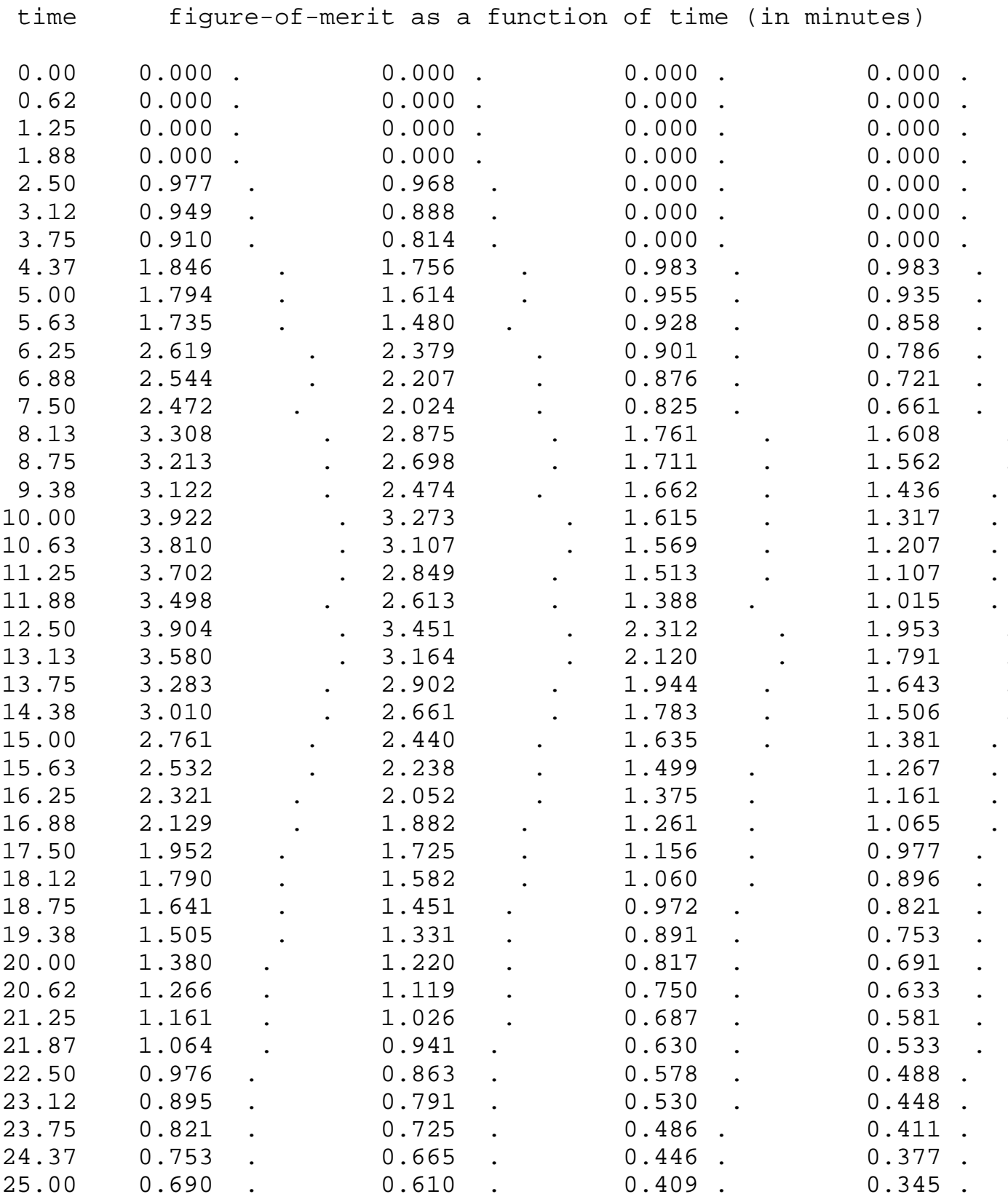

Figure 3: Some fairly long route flap cycles, repeated for 12 minutes, followed by a period of stability. 
The reuse list is used to provide a means of fast evaluation of route that had been suppressed, but had been stable long enough to be reused again or had been suppressed long enough that it can be treated as a new route. The following two operations are described.

1. Inserting into a reuse list (Section 4.8.6)

2. Reuse list processing every delta-t seconds (Section 4.8.7)

\subsubsection{Processing a New Peer or New Routes}

When a peer comes up, no action is required if the routes had no previous history of instability, for example if this is the first time the peer is coming up and announcing these routes. For each route, the pointer to the damping structure would be zeroed and route used. The same action is taken for a new route or a route that has been down long enough that the figure of merit reached zero and the damping structure was deleted.

\subsubsection{Processing Unreachable Messages}

When a route is withdrawn or changed (Section 4.8 .4 describes how a change is handled), the following procedure is used.

If there is no previous stability history (the damping structure pointer is zero), then:

1. allocate a damping structure

2. set figure-of-merit $=1$

3. withdraw the route

Otherwise, if there is an existing damping structure, then:

1. set $t$-diff $=t$-now $-t$-updated

2. if (t-diff puts you off the end of the array) \{ setfigure-of-merit $=1$

\}else \{

setfigure-of-merit =figure-of-merit *decay-array-ok [t-diff ]+ 1 if(figure-of-merit >ceiling) \{ setfigure-of-merit $=$ ceiling 


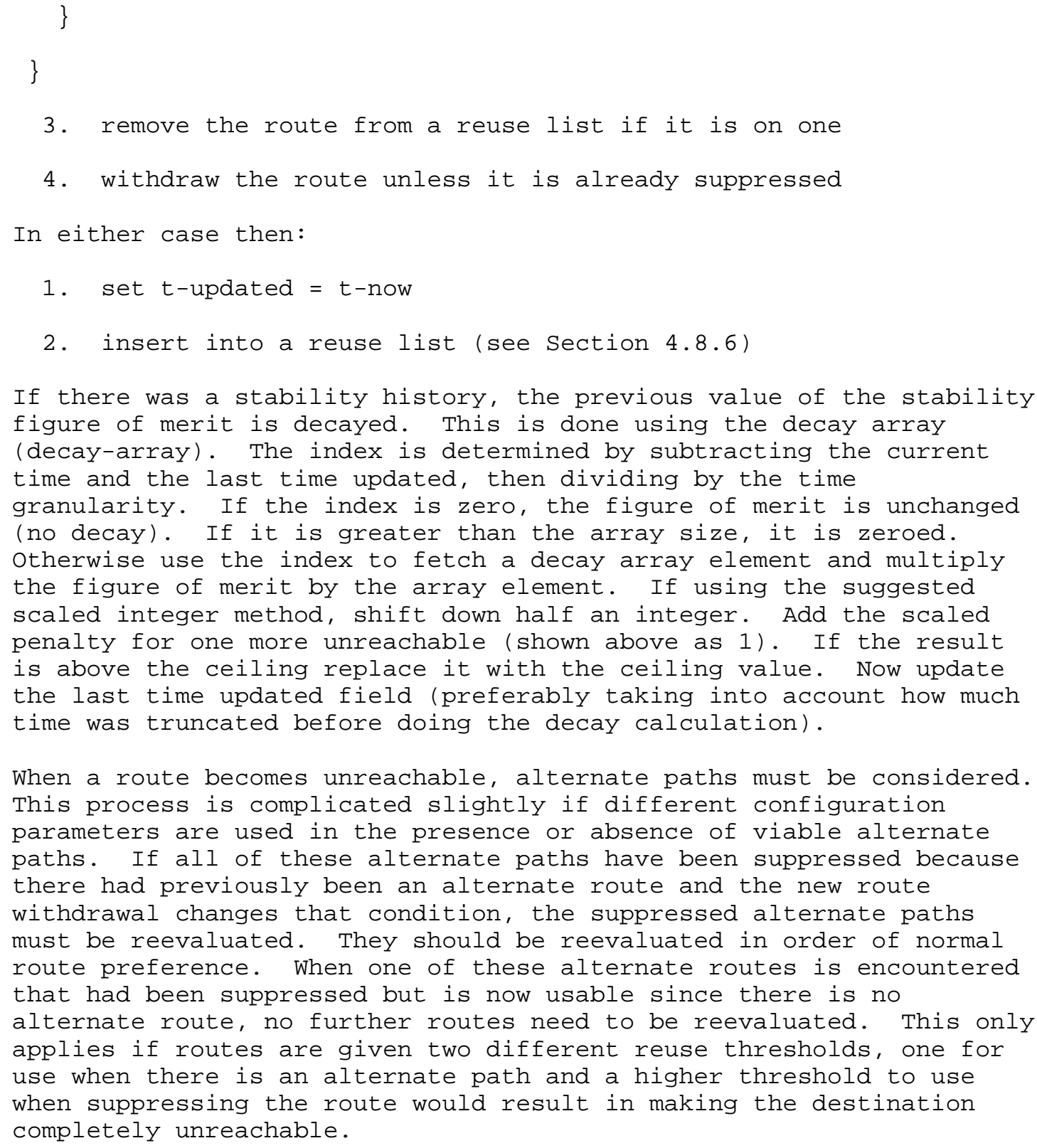


1. don't create a new damping structure

2. use the route

If an damping structure exists, the figure of merit is decayed and the figure of merit and last time updated fields are updated. A decision is now made as to whether the route can be used immediately or needs to be suppressed for some period of time.

1. set $t$-diff $=t$-now $-t$-updated

2. if (t-diff puts you off the end of the array) \{ set figure-of-merit $=0$

\}else \{

set figure-of-merit= figure-of-merit* decay-array-ng[t-diff] \}

3. if ( not suppressed and figure-of-merit < cut ) \{ use the route \}else if( suppressed and figure-of-merit< reuse) \{ set state tonot suppressed

4. if (figure-of-merit >0) \{ set $t$-updated $=t$-now \}else \{ 


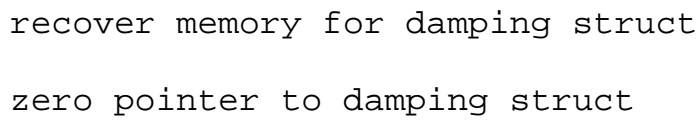

If the route is deemed usable, a search for the current best route must be made. The newly reachable route is then evaluated according to the BGP protocol rules for route selection.

If the new route is usable, the previous best route is examined. Prior to route comparisons, the current best route may have to be reevaluated if separate parameter sets are used depending on the presence or absence of an alternate route. If there had been no alternate the previous best route may be suppressed.

If the new route is to be suppressed it is placed on a reuse list only if it would have been preferred to the current best route had the new route been accepted as stable. There is no reason to queue a route on a reuse list if after the route becomes usable it would not be used anyway due to the existence of a more preferred route. Such a route would not have to be reevaluated unless the preferred route became unreachable. As specified here, the less preferred route would be reevaluated and potentially used or potentially added to a reuse list when processing the withdrawal of a more preferred best route.

\subsubsection{Processing Route Changes}

If a route is replaced by a peer router by supplying a new path, the route that is being replaced should be treated as if an unreachable were received (see Section 4.8.2). This will occur when a peer somewhere back in the AS path is continuously switching between two AS paths and that peer is not damping route flap (or applying less damping). There is no way to determine if one AS path is stable and the other is flapping, or if they are both flapping. If the cycle is sufficiently short compared to convergence times neither route through that peer will deliver packets very reliably. Since there is no way to affect the peer such that it chooses the stable of the two AS paths, the only viable option is to penalize both routes by considering each change as an unreachable followed by a route advertisement.

\subsubsection{Processing A Peer Router Loss}

When a peer routing session is broken, either all individual routes advertised by that peer may be marked as unstable, or the peering session itself may be marked as unstable. Marking the peer will save 
considerable memory. Since the individual routes are advertised as unreachable to routers beyond the immediate problem, per route state will be incurred beyond the peer immediately adjacent to the BGP session that went down. If the instability continues, the immediately adjacent router need only keep track of the peer stability history. The routers beyond that point will receive no further advertisements or withdrawal of routes and will dispose of the damping structure over time.

BGP notification through an optional transitive attribute that damping will already be applied may be considered in the future to reduce the number of routers that incur damping structure storage overhead.

4.8.6 Inserting into the Reuse Timer List

The reuse lists are used to provide a means of fast evaluation of route that had been suppressed, but had been stable long enough to be reused again. The data structure consists of a series of list heads. Each list contains a set of routes that are scheduled for reevaluation at approximately the same time. The set of reuse list heads are treated as a circular array. Refer to Figure 4.

A simple implementation of the circular array of list heads would be an array containing the list heads. An offset is used when accessing the array. The offset would identify the first list. The Nth list would be at the index corresponding to $\mathrm{N}$ plus the offset modulo the number of list heads. This design will be assumed in the examples that follow.

A key requirement is to be able to insert an entry in the most appropriate queue with a minimum of computation. The computation is given only the current value of figure-of-merit. Instead of a computation which would involve a logarithm, the reuse array (reusearray[]) described in section 4.6 is used. The array, scale, and bounds are precomputed to map figure-of-merit to the nearest list head without requiring a logarithm to be computed (see Section 4.5). 


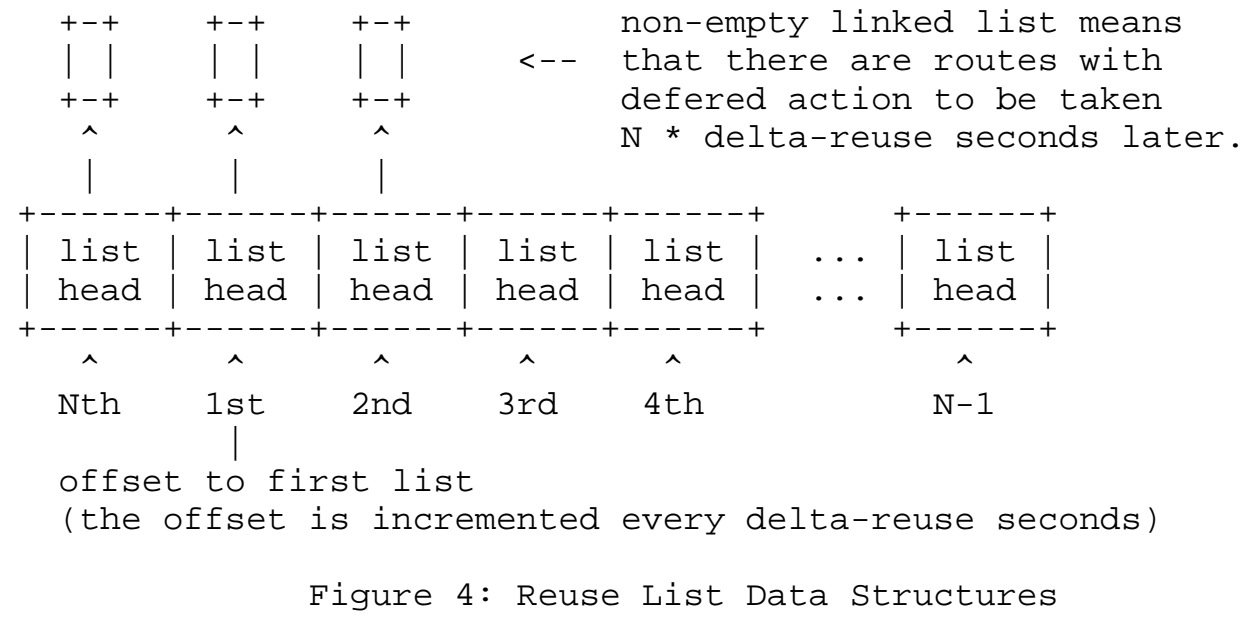

Note that in the following sections the operator prefix notation "modulo a b" means "b $\%$ a" in C language algebraic operator notation. For example, "modulo 16 1023" would be 15 .

1. scale figure-of-merit for the index array lookup producing index

2. check index against the array bound

3. if (within the array bound) \{ set index =reuse-array [index ]

\}else \{ set index $=$ reuse-list-size -1

\}

4. insert into the list reuse-list [ moduloreuse-list-size (index toffset)]

Choosing the correct reuse list involves only a multiply and shift to do the scaling, an integer truncation, then an array lookup in the reuse array (reuse-array[]). The value retrieved from the reuse array is used to select a reuse list. The reuse list is a circular list. The most common method of implementing a circular list is to use an array and apply an offset and modulo operation to pick the correct array entry. The offset is incremented to rotate the circular list. 


\subsubsection{Handling Reuse Timer Events}

The granularity of the reuse timer should be more coarse than that of the decay timer. As a result, when the reuse timer fires, suppressed routes should be decayed by multiple increments of decay time. Some computation can be avoided by always inserting into the reuse list corresponding to one time increment past reuse eligibility. In cases where the reuse lists have a longer "memory" than the "decay memory" (described above), all of the routes in the first queue will be available for immediate reuse if reachable or the history entry could be disposed of if unreachable.

When it is time to advance the lists, the first queue on the reuse list must be processed and the circular queue must be rotated. Using an array and an offset as a circular array (as described in section 4.8.6), the algorithm below is repeated every delta-reuse seconds.

1. save a pointer to the current zeroth queue head and zero the list head entry

2. set offset $=$ modulo reuse-list-size ( offset +1 ), thereby rotating the circular queue of list-heads

3. if ( the saved list head pointer is non-empty )

for each entry \{

sett-diff $=t-$ now $-t$-updated

set figure-of-merit =figure-of-merit *decay-array-ok [t-diff ]

sett-updated $=$ - - now

if ( figure-of-merit< reuse)

reuse the route

else

re-insert into another list (seesection 4.8.6)

\}

The value of the zeroth list head would be saved and the array entry itself zeroed. The list heads would then be advanced by incrementing the offset. Starting with the saved head of the old zeroth list, each route would be reevaluated and used, disposed of entirely or requeued if it were not ready for reuse. If a route is used, it must 
be treated as if it were a new route advertisement as described in Section 4.8 .3 .

5 Implementation Experience

The first implementations of "route flap damping" were the route server daemon (rsd) coding by Ramesh Govindan (ISI) and the Cisco IOS implementation by Ravi Chandra. Both implementations first became available in 1995 and have been used extensively. The rsd implementation has been in use in route servers at the NSF funded Network Access Points (NAPs) and at other major Internet interconnects. The Cisco IOS version has been in use by Internet Service Providers worldwide. The rsd implementation has been integrated in releases of gated (see http://www.gated.org) and is available in commercial routers using gated.

There are now more than 2 years of BGP route damping deployment experience. Some problems have occurred in deployment. So far these are solvable by careful implementation of the algorithm and by careful deployment. In some topologies coordinated deployment can be helpful and in all cases disclosure of the use of route damping and the parameters used is highly beneficial in debugging connectivity problems.

Some of the problems have occurred due to subtle implementation errors. Route damping should never be applied on IBGP learned routes. To do so can open the possibility for persistent route loops. When IBGP routes within an AS are inconsistent, route loops can easily form. Suppressing IBGP learned routes causes such inconsistencies. Implementations should disallow configuration of route damping on IBGP peers.

Penalties for instability should only be applied when a route is removed or replaced and not when a route is added. If damping parameters are applied consistently, this implementation constraint will result in a stable secondary path being preferred over an unstable primary path due to damping of the primary path near the source.

In topologies where multiple AS paths to a given destination exist flapping of the primary path can result in suppression of the secondary path. This can occur if no damping is being done near the cause of the route flap or if damping is being applied more aggressively by a distant AS. This problem can be solved in one of two ways. Damping can be done near the source of the route flap and the damping parameters can be made consistent. Alternately, a distant AS which insists on more aggressive damping parameters can disable penalizing routes on AS path change, penalizing routes only 
if they are withdrawn completely. In order to do so, the implementation must support this option (as described in section $4.4 .3)$.

Route flap should be damped near the source. Single homed destinations can be covered by static routes. Aggregation provides another means of damping. Providers should damp their own internal problems, however damping on IGP link state origination is not yet implemented by router vendors. Providers which use multiple AS within their own topology should damp between their own AS. Providers should damp adjacent providers AS.

Damping provides a means to limit propagation excessive route change when connectivity is highly intermittent. Once a problem is corrected, damping state corresponding to the prefixes known to be damped due to the problem just fixed can be manually cleared. In order to determine where damping may have occurred after connectivity problems, providers should publish their damping parameters. Providers should be willing to manually clear damping on specific prefixes or AS paths at the request of other providers when the request is accompanied by credible assurance that the problem has truly been addressed.

By damping their own routing information, providers can reduce their own need to make requests of other providers to clear damping state after correcting a problem. Providers should be pro-active and monitor what prefixes and paths are suppressed in addition to monitoring link states and BGP session state.

\section{Acknowledgements}

This work and this document may not have been completed without the advise, comments and encouragement of Yakov Rekhter (Cisco). Dennis Ferguson (MCI) provided a description of the algorithms in the gated BGP implementation and many valuable comments and insights. David Bolen (ANS) and Jordan Becker (ANS) provided valuable comments, particularly regarding early simulations. Over four years elapsed between the initial draft presented to the BGP WG (October 1993) and this iteration. At the time of this writing there is significant experience with two implementations, each having been deployed since 1995. One was led by Ramesh Govindan (ISI) for the NSF Routing Arbiter project. The second was led by Ravi Chandra (Cisco). Sean Doran (Sprintlink) and Serpil Bayraktar (ANS) were among the early independent testers of the Cisco pre-beta implementation. Valuable comments and implementation feedback were shared by many individuals on the IETF IDR WG and the RIPE Routing Work Group and in NANOG and IEPG. 
Thanks also to Rob Coltun (Fore Systems), Sanjay Wadhwa (Fore), John Scudder (IENG), Eric Bennet (IENG) and Jayesh Bhatt (Bay Networks) for pointing out errors in the math uncovered during coding of more recent implementations. These errors appeared in the details of the implementation suggestion sections written after the first two implementations were completed. Thanks also to Vern Paxson for a very thorough review resulting in numerous clarifications to the document.

References

[1] Gross, P., and Y. Rekhter, "Application of the border gateway protocol in the internet", RFC 1268, October 1991.

[2] ISO/IEC. Iso/iec 10747 - information technology - telecommunications and information exchange between systems - protocol for exchange of inter-domain routeing information among intermediate systems to support forwarding of iso 8473 pdus. Technical report, International Organization for Standardization, August 1994. ftp://merit.edu/pub/iso/idrp.ps.gz.

[3] Lougheed, K., and Y. Rekhter, "A border gateway protocol 3 (BGP3) ", RFC 1267, October 1991.

[4] Rekhter, Y., and P. Gross, "Application of the border gateway protocol in the internet", RFC 1772, March 1995.

[5] Rekhter, Y., and T. Li, "A border gateway protocol 4 (BGP-4)", RFC 1771, March 1995.

[6] Rekhter, Y., and C. Topolcic,"Exchanging routing information across provider boundaries in the CIDR environment", RFC 1520, September 1993 .

[7] Traina, P., "BGP-4 protocol analysis", RFC 1774, March 1995.

[8] Traina, P., "Experience with the BGP-4 protocol", RFC 1773, March 1995.

Security Considerations

The practices outlined in this document do not further weaken the security of the routing protocols. Denial of service is possible in an already insecure routing environment but these practices only contribute to the persistence of such attacks and do not impact the methods of prevention and the methods of determining the source. 


\author{
Authors' Addresses \\ Curtis Villamizar \\ ANS \\ EMail: curtisdans.net \\ Ravi Chandra \\ Cisco Systems \\ EMail: rchandra@cisco.com \\ Ramesh Govindan \\ ISI \\ EMail: govindandisi.edu
}


Full Copyright statement

Copyright (C) The Internet Society (1998). All Rights Reserved.

This document and translations of it may be copied and furnished to others, and derivative works that comment on or otherwise explain it or assist in its implementation may be prepared, copied, published and distributed, in whole or in part, without restriction of any kind, provided that the above copyright notice and this paragraph are included on all such copies and derivative works. However, this document itself may not be modified in any way, such as by removing the copyright notice or references to the Internet society or other Internet organizations, except as needed for the purpose of developing Internet standards in which case the procedures for copyrights defined in the Internet Standards process must be followed, or as required to translate it into languages other than English.

The limited permissions granted above are perpetual and will not be revoked by the Internet society or its successors or assigns.

This document and the information contained herein is provided on an "AS IS" basis and THE INTERNET SOCIETY AND THE INTERNET ENGINEERING TASK FORCE DISCLAIMS ALL WARRANTIES, EXPRESS OR IMPLIED, INCLUDING BUT NOT LIMITED TO ANY WARRANTY THAT THE USE OF THE INFORMATION HEREIN WILL NOT INFRINGE ANY RIGHTS OR ANY IMPLIED WARRANTIES OF MERCHANTABILITY OR FITNESS FOR A PARTICULAR PURPOSE. 